\title{
Use of Modelica language to model an MV compensated electrical network and its protection equipment : comparison with EMTP
}

\author{
Olivier Chilard \\ Jean-Philippe Tavella \\ Olivier Devaux \\ EDF Research and Development \\ 1, avenue du général de Gaulle, 92140 Clamart France \\ olivier.chilard@edf.fr jean-philippe.tavella@edf.fr olivier.devaux@edf.fr
}

\begin{abstract}
Today, neutral compensation based on Petersen coils is being applied in many MV electrical networks. The main reasons for this are :

- an important expansion of the underground network in rural environment. This leads to high phase-to-ground capacities of the outgoing feeders, which increases fault currents in networks using impedant grounding,

- an increased sensitivity of the customers to the quality of supply,

- changes in international standards (insulation coordination).
\end{abstract}

The introduction of arc suppression Petersen coils allows both to reduce the current in single phase-toground faults and to improve the quality of supply by reducing short supply disconnection.

Adapting this solution to the existing networks made it necessary to change the protection system, by using zero sequence wattmetric relays.

The feedbacks of the zero sequence wattmetric relays operating today show the need of an evolution of this protection equipment specifications. To address this need, EDF R\&D has investigated the use of Modelica language for the electrical system modeling.

At first the relevance of Modelica language for electrical systems modeling has been studied. This work was made from a comparison of simulation results with those traditionally obtained with EMTP software, normally used by EDF R\&D. This paper details this approach and underlines the interest of Modelica for the electrical network fields. The next step will be the use of the ModelicaML profile in order to establish a new version of the protection system specification.

\section{Introduction}

Currently, in order to design new control devices such as protection equipment, Matlab and EMTP are widely used. The solutions obtained are then validated on the field. Once a final solution is retained, a specification paper is then written and sent to the manufacturers which provide industrial solutions.

However, paper specifications are not formal, thus they may be interpreted differently by manufacturers and lead to some difficulties in conception phases. Moreover, when an equipment has been in operation for several years, if the initial solution has to be upgraded, the initial specification paper might be difficult to exploit again. Indeed, the informal description of the expected equipments often leads to a paper with redundant, missing or interpretable requirements.

In this context, a new approach based on the Modelica language has been studied. Modelica has been applied first in the particular case of the zero sequence wattmetric relay used in MV compensated networks. Moreover, this approach is intended to be used both for the development and specification phases.

This paper presents the modeling work and gives a comparison with a more traditional approach using EMTP-RV [3]. The simulation results are also provided and compared.

The final objective will be to provide a formal executable specification of the expected equipment. 


\section{Description of the use case retained}

The use case refers to the modeling and the simulation of two zero sequence wattmetric protections and a simplified MV compensated network. The protection is described in a specification paper established by EDF R\&D. It has been developed to protect MV neutral compensated networks against mean and medium resistive single phase faults. This protection denoted $\mathrm{PWH}$, is based on the analysis of both transient and steady state values of residual current and residual voltage available in the compensated MV substation.

Let's briefly recall the principle of a compensated network and that of the PWH.

In rural MV distribution networks, each feeder is mainly constituted of an overhead line and thus, when a fault occurs, the phase-to-ground capacitance current values are low (Figure 1). Therefore in these cases the neutral of the HV/MV transformer is grounded through a resistor and the fault current is equal to the neutral current.

Figure 1 : Principles of compensated networks

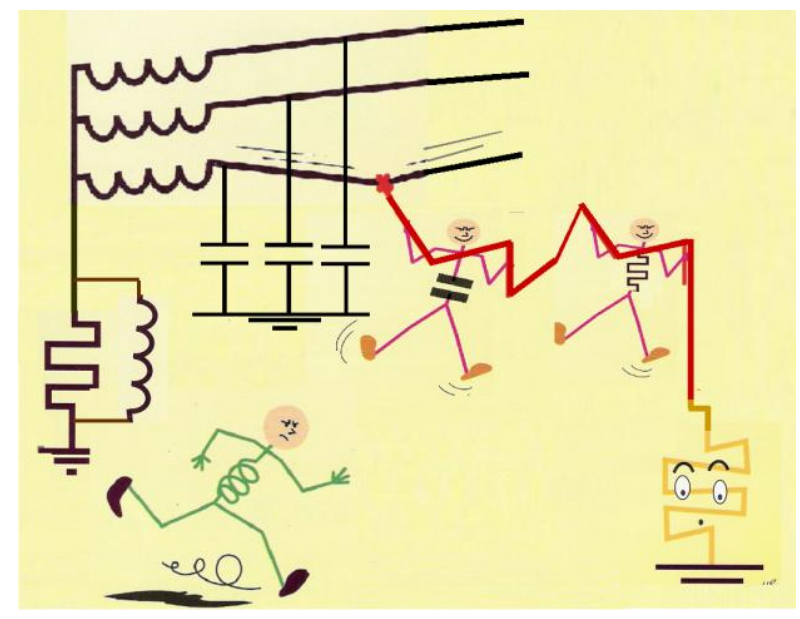

In mixed MV Distribution networks, an important part of each outgoing feeder is composed of underground cables. Therefore, contrary to the previous case, when a single fault occurs, the total capacitor current value seen at the MV busbar is important. Thus the resistor of the MV neutral grounding is replaced by a coil (a resistor in parallel with an inductor, refer to Figure 1) in order to compensate the zero sequence capacitor current and thus limit the current according to the international standard (insulation coordination). Consequently, if the mismatch of the coil is adjusted to zero Amps, the fault current is limited to the active neutral current part of the coil.
Concerning the network modeling, a simplified MV compensated network is considered. The following assumptions are made :

- The single phase fault is considered close to the MV busbar. Thus, the reactance of each outgoing feeder is neglected and only the phase-to-ground capacitance is considered,

- The MV substation three phase voltage source is assumed to be balanced,

- Consumption and reactive power compensators are not considered.

\section{Figure 2 : MV compensated network retained}

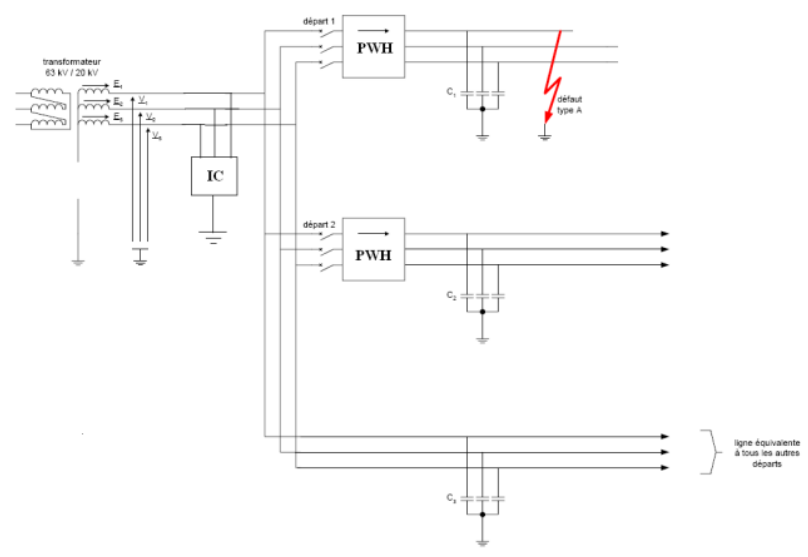

Therefore, the simplified MV network scheme retained is given in Figure 2. The substation is defined by its HV/MV transformer, its coil inserted on the neutral grounding and its MV busbar. One faulty feeder and two unfaulty feeders are considered. The second unfaulty feeder (without protection) represents an aggregation of all other unfaulty feeders. Two PWHs are also considered and respectively allocated to the faulty feeder and to the first unfaulty feeder (Figure 2). Its aim is to eliminate the single phase fault. To do that, the PWH has to send a trip to the circuit breaker of the faulty feeder.

A PWH is based on the analysis of both transient and steady state values of residual current and residual voltage measured at the MV substation.

PWH selective action is based on the known fact that the active component of the residual current in the faulty feeder is of the opposite direction and much greater than on any of the unfaulty feeders. Therefore, on the faulty feeder, the zero-sequence wattmetric relays detects negative zero sequence active power. On contrary, the protection placed on the unfaulty feeder detects a positive zero sequence active power. 
The PWH protection has to protect the network against two types of phase-to-ground faults :

- A permanent fault which involves residual current with a preponderant component at $50 \mathrm{~Hz}$, after the transient phenomena due to the occurrence of the fault,

- A series of self-extinguishing faults, which is considered as the same fault.

In order to detect a permanent fault, the RMS values of the residual voltage $\&$ current and the residual active \& reactive power are calculated for a cycle of $20 \mathrm{~ms}$. For the two types of faults, residual power mean value is also calculated for a cycle of 60 ms (Figure 3).

Depending on the results of these calculations, according to a condition test, a positive or negative residual power message is send to the logical part of the protection which elaborates a trip protection message toward the circuit breaker of the faulty feeder.

Figure 3 : Principles of the PWH

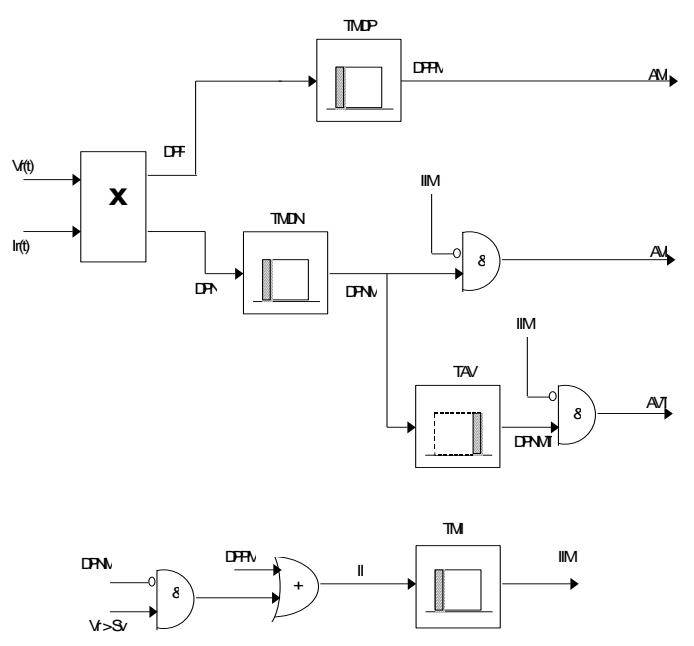

\section{Overview of the use case model im- plemented with EMTP-RV}

\subsection{Overview of EMTP-RV}

EMTP-RV (ElectroMagnetic Transient Program) is a well-known software used for the simulation analysis of electrical power system.

The program is meant for solving problems such as :

- Switching transients and overvoltages,

- Short term analysis of disturbances,

- Overcurrent calculations,

- Control of electric drives,

- FACTS

\subsection{Use case modeling}

\subsubsection{EMTP Modeling approaches}

Two types of components can be used under EMTP : traditional electrical components for electrical circuits and block diagrams for the description of automatic control of electrical systems. Most of the electrical components are not modifiable by users. Thus, if a new component is to be implemented, the user has the option of building it with block diagrams and elementary electrical components.

Another possibility is to compile a DLL or an Sfunction. The DLL approach consists of a causal description of the component according to the numeric solver of EMTP-RV. Thus, the DLL is a discretization of the mathematical representation of the component written in $\mathrm{C}$ language.

The S-function can be, for instance, provided by a model previously established under Simulink or Dymola tools. With this feature the numeric solver of the model is included in the S-function and can be different from EMTP's.

\subsubsection{Use case modeling}

The MV compensated network model implementation is immediate. A predefined RL coupled impedance has been used in order to respectively define :

- The resistor and inductor (in parallel) of the neutral grounding impedance,

- The short circuit impedance of the three phase voltage source.

These components represent an equivalent model of both the transformer and the neutral impedance connected in its MV terminals (Figure 2). To do so, the positive, negative and zero sequence parameters are chosen for each element.

A simple capacitor grounding has been placed at each phase of each feeder. 
Figure 4 : The use case implementation under EMTP
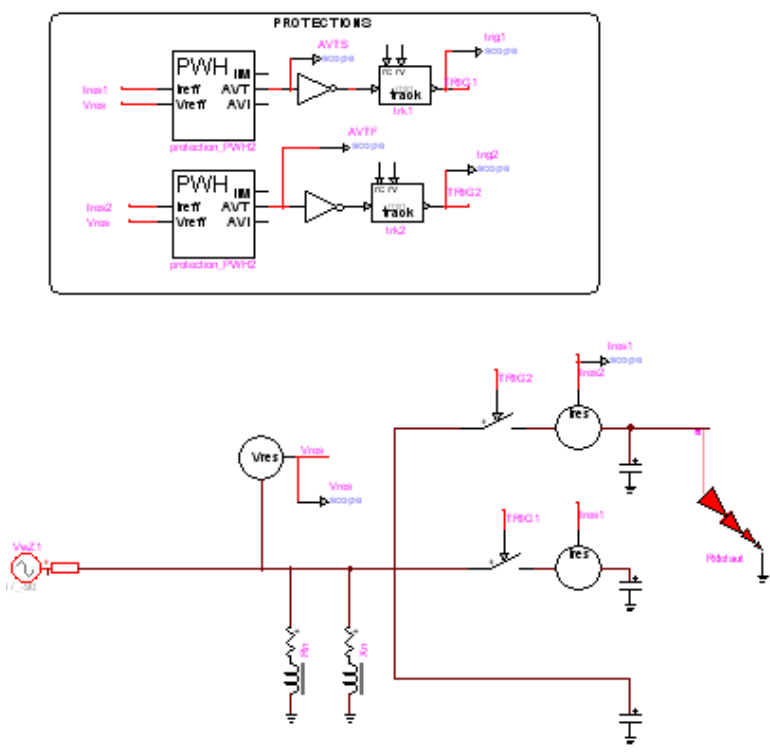

For the zero sequence wattmetric relays implementation a combination of different block diagrams has been used in order to model the PWHs. The resulting model, derived from the structure given in Figure 3 is shown in Figure 5.

\section{Figure 5 : PWH model under EMTP}

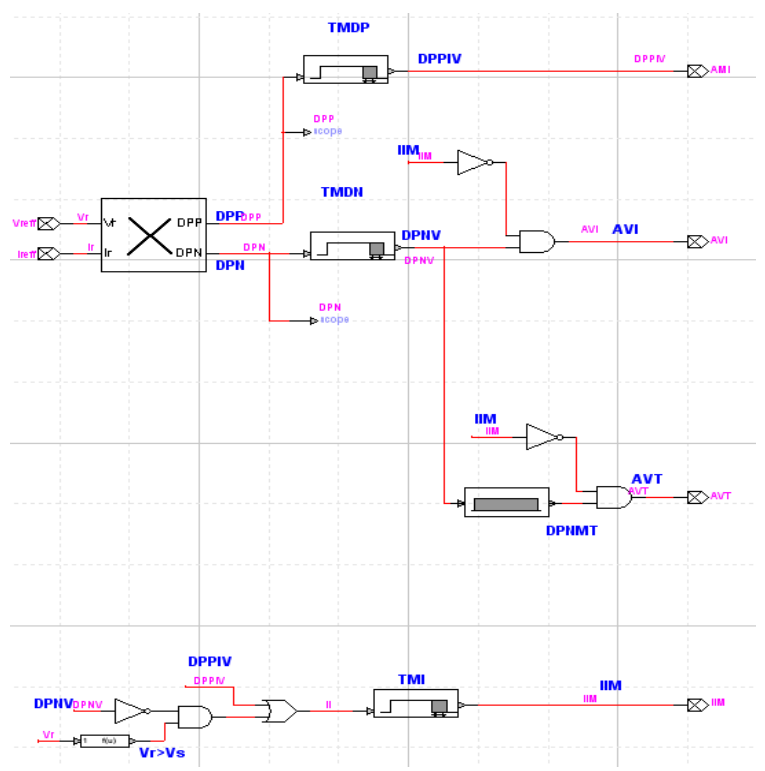

\section{Overview of the use case model im- plemented with the Modelica lan- guage}

Like under EMTP-RV, the simplified MV compensated network model implementation is immediate with Modelica. To do so, the components inside the official "Modelica.Electrical.Multiphase" package provided with the language have been used.
In order to simulate the use case, the Dymola tool based on the Modelica language has been chosen. It provides the numeric solvers required for the simulations and allows a graphical representation of the model.

Figure 6 : Use case implementation with Modelica

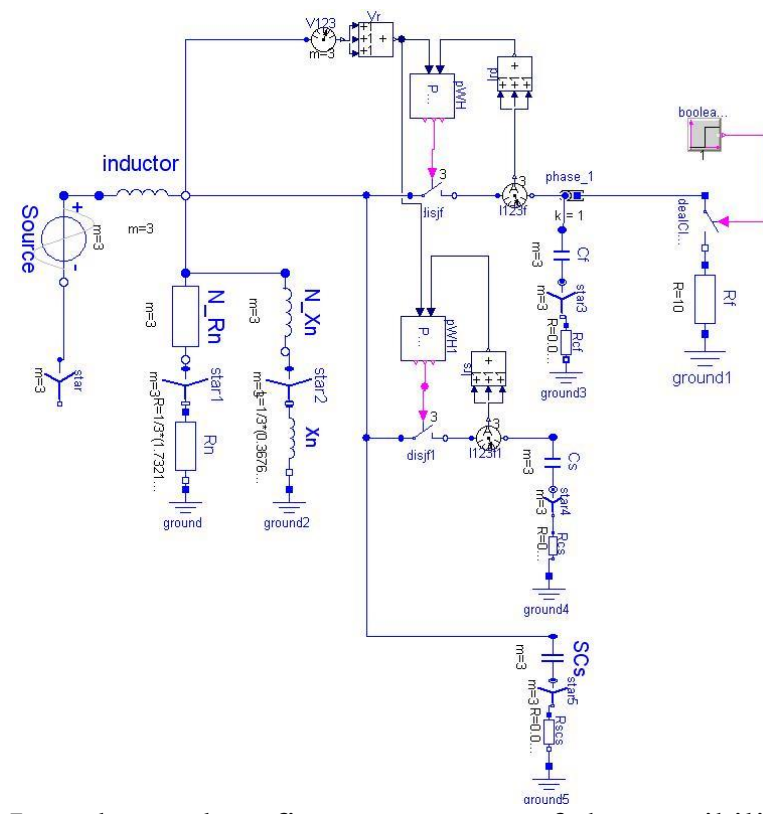

In order to do a first assessment of the possibilities provided by the "Modelica.Electrical.Machines" package, an alternative model with a transformer has been implemented too (Figure 7).

Figure 7 : Alternative modeling with a transformer

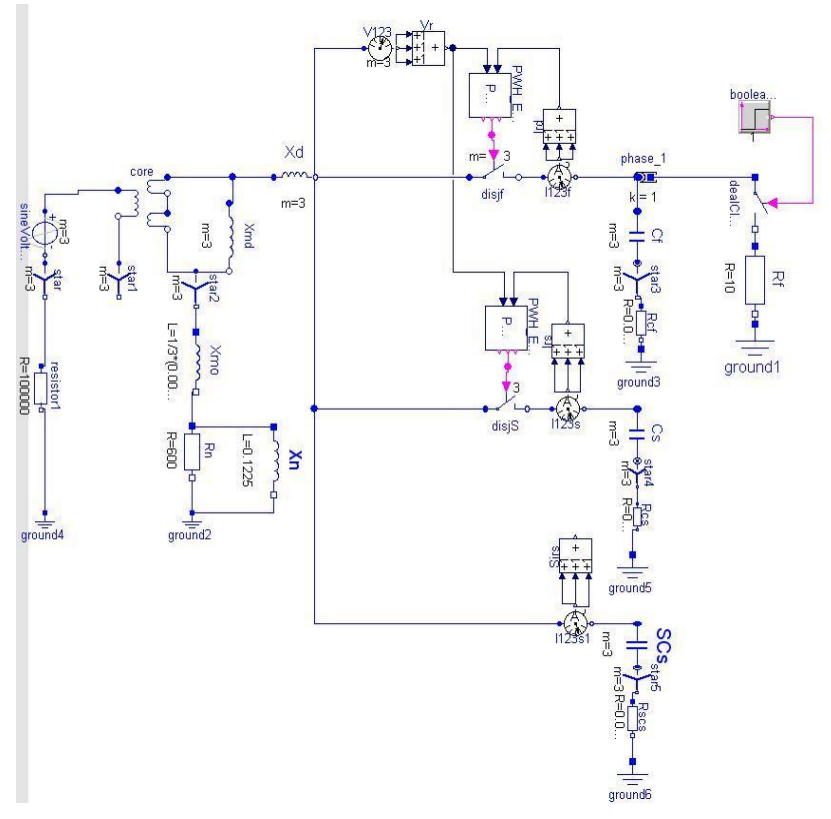


It is denoted that the RL coupled component is not available in the Modelica libraries. Thus, the Rn, $\mathrm{Xn}$ and Xmo given in Figure 6 and Figure 7 are defined as $1 / 3$ of the difference between the zero sequence and positive sequence impedances expected with the positive sequence impedance placed in each phase (Figure 6 : N_Rn, N_Xn / Figure 7 : Xmd).

In order to assess the Modelica language possibilities, the distribution MV Network has been also implemented without the use of the Modelica "Modelica.Electrical.Multiphase" package (Figure 8). In this case, coupled components have been created. To do so, the voltage and current variables of each connector are defined as a column vector with three elements belonging to each phase (Figure 9). Thus, each component has been defined by inheritance and from matrix equations (Figure 10).

Like EMTP-RV, zero sequence and positive sequence input parameters are given for each of these components.

Figure 8 : Use case implementation without the use of the "Modelica.Electrical.Multiphase" package

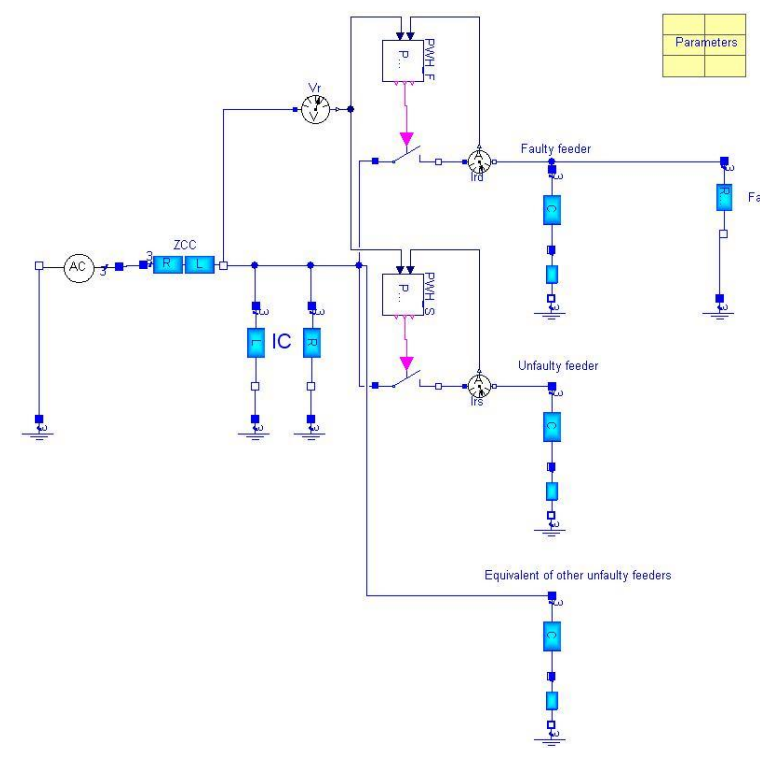

Figure 9 : Example of the 'two pin' partial model created without any standard component

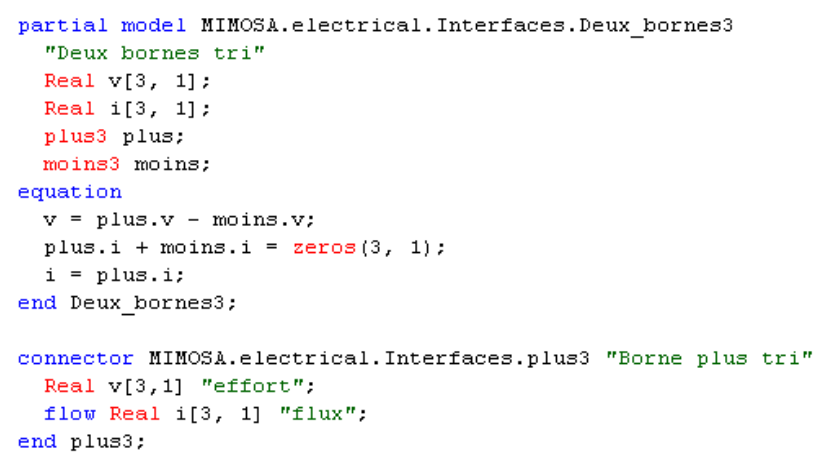

\section{Figure 10 : Inductor $L$ coupled model}

$\mathrm{Xd}$ and Xo respectively correspond to the positive and zero sequence impedances. The negative sequence impedance is taken equal to the positive one.

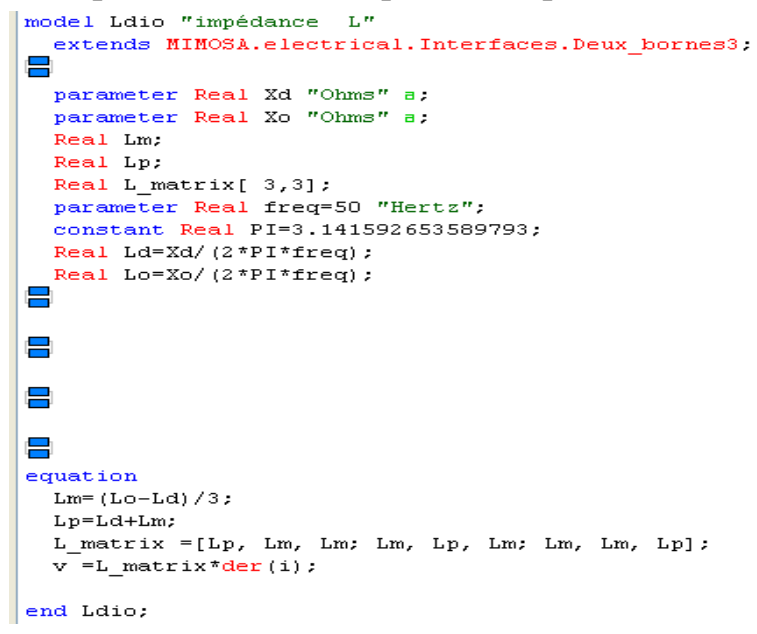

The three previously described Modelica models (with and without "Modelica.Electrical.Multiphase" package components) are equivalent as showed by the simulation results obtained in Figure 11.

Figure 11 : Fault current curves from the three models

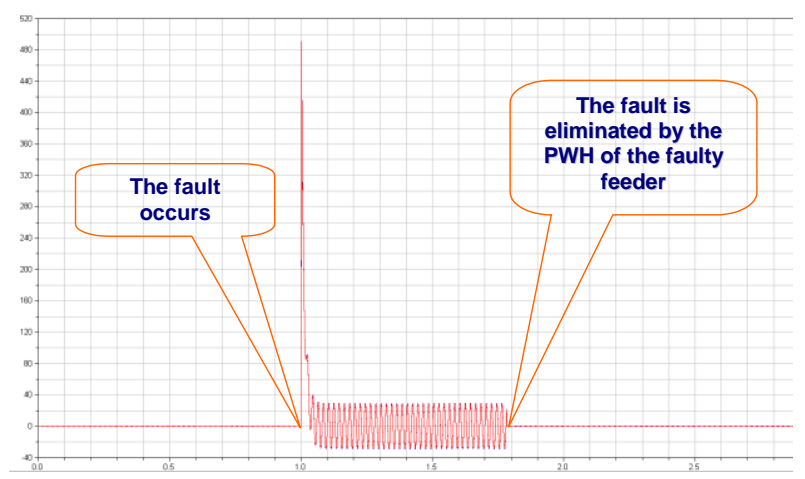

For the zero sequence wattmetric relays implementation, a combination of the different block diagrams has been used in order to model the PWHs. However, these blocks have been created with the Modelica language in order to facilitate this work. The resulting model is presented in the Figure 12. 
Figure 12 : PWH model under Modelica/Dymola

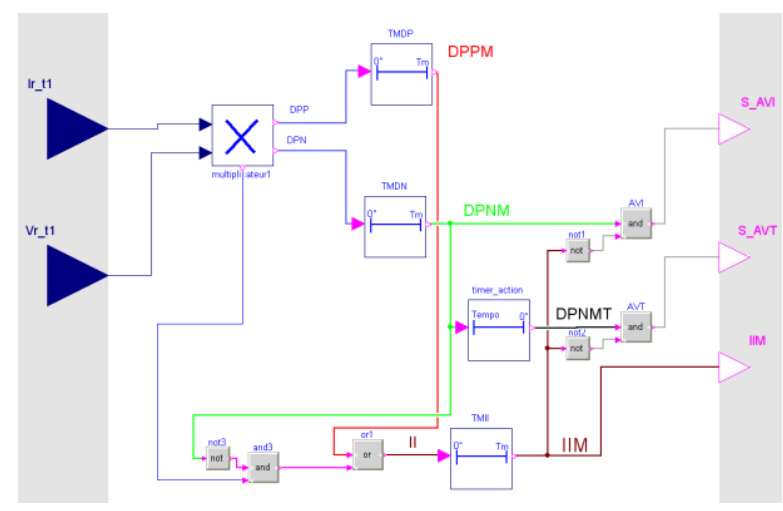

\section{Modelica and EMTP}

\subsection{Validation of Modelica modeling}

Some simulations have been done in order to validate the model developed with the Modelica language.

To do so, EMTP has been considered as the benchmark tool.

Under Dymola and EMTP, all the electrical signals belonging respectively to the PWH and the MV network are identical. In this paper, only the fault current is presented (Figure 13).

It is obtained from simulations performed on models given in Figure 4 and Figure 8. The fault current values are identical such as the fault clearance time due the PWH action.

Figure 13 : Phase-to-ground fault current Blue : EMTP Red : Modelica/Dymola

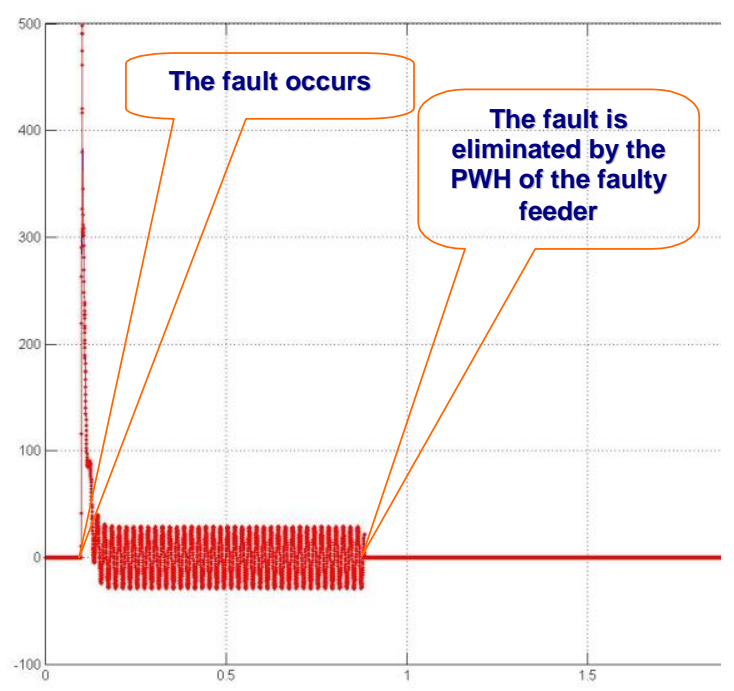

\subsection{Modelica and EMTP modeling comparison}

The Modelica model, with all components created by the user (Figure 8) is compared with the EMTP model (Figure 4). EMTP has, for the electrical power part, implicit components and, for the control command part, elementary explicit blocks. These components are not modifiable and thereby to develop new components, the user has to :

- Either create more or less difficult elementary components associations,

- Or build a DLL with S-function.

For the use case considered in this paper, only explicit and implicit blocks were used. Thus, given the basic components available in EMTP, some tricks have been used to model the treatment of the multiplier outputs named DPN and DPP (Figure 14). From this very simple case, it is not difficult to understand that it would be very difficult to implement and verify more complex systems such as distance protections or advanced functions developed in the SmartGrid. Although this may not be unfeasible (to be validated according to the available basic blocks), these developments would be difficult without any guarantee of success. Moreover, the level of readability of such models would be bad and this approach leads to an increase of the number of variables and thus unnecessarily constraints the solver.

In the opposite, Modelica models are simpler because the different blocks of the explicit PWH can be defined by the user. In other words, rather than associating basic blocks of the native library of Modeli$\mathrm{ca}$, the user can create more adapted components or modify some existing blocks. Moreover, the Modelica language provides all specific language paradigms for algorithm developments and so, unlike EMTP, statements related to the treatment of the multiplier are close to the description given in the PWH specification paper (Figure 14 and Figure 15). 
Figure 14 : Treatment of the PWH multiplier outputs (DPP \& DPN)
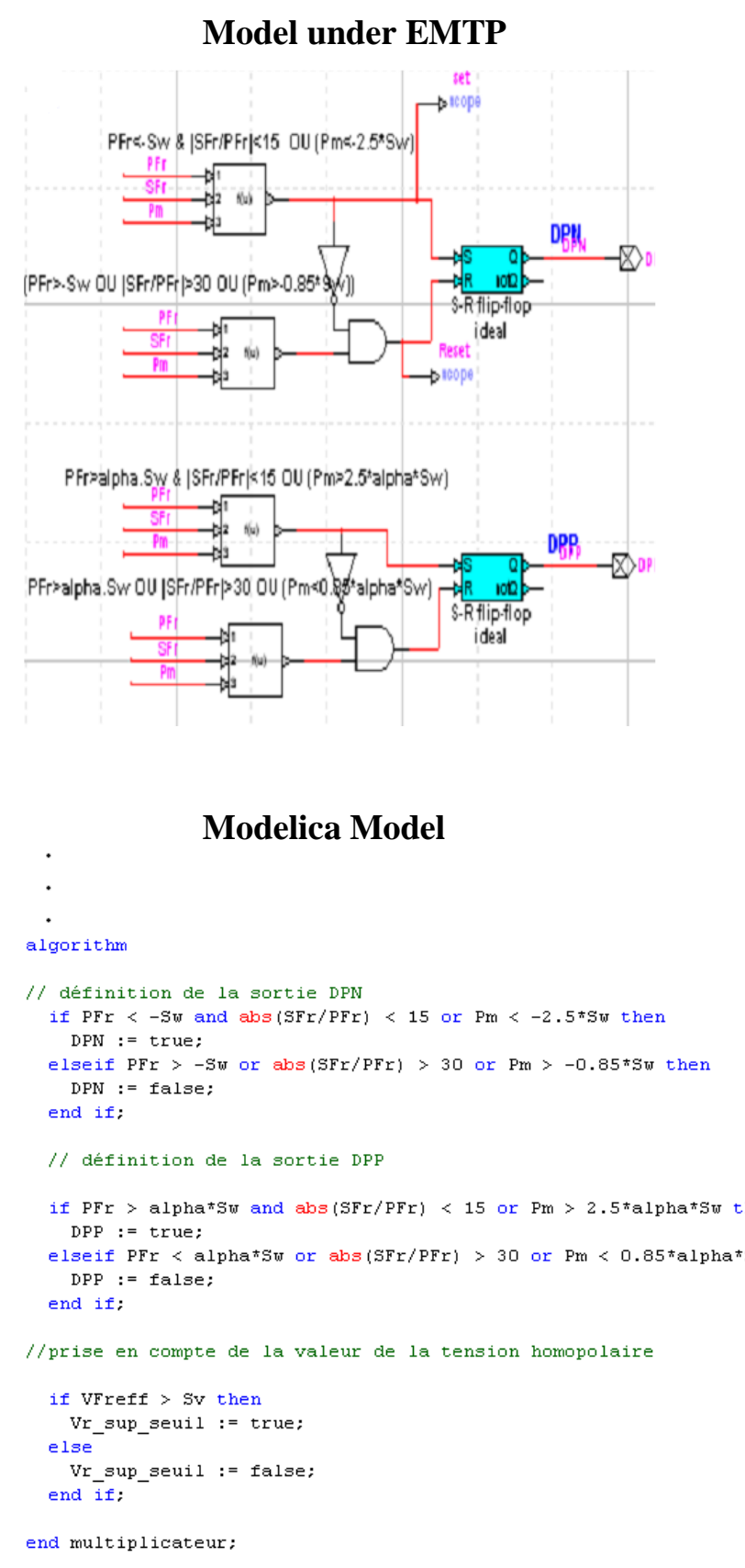

Figure 15 : PWH specification extract focused on the processing of the multiplier outputs DPN \& DPP

- $\underline{\text { DNN (Détection Puissance Négative) }}$ $\mathrm{DPN}=1$ si $\quad\left(\mathrm{PF}_{\mathrm{r}}<-\mathrm{SW}\right)$ et $(|\mathrm{SFr} / \mathrm{PFr}|<15)$ $\mathrm{DPN}=0$ si $\left(\mathrm{PF}_{\mathrm{r}}>-\mathrm{SW}\right)$ ou $(|\mathrm{SFr} / \mathrm{PFr}|>30)$

- $\underline{\text { DPP }}$ (Détection Puissance Positive) $\mathrm{DPP}=1$ si $\left(\mathrm{PF}_{\mathrm{r}}>\alpha \mathrm{SW}\right)$ et $(|\mathrm{SFr} / \mathrm{PFr}|<15)$ $\mathrm{DPP}=0$ si $\left(\mathrm{PF}_{\mathrm{r}}<\alpha \mathrm{SW}\right)$ ou $(|\mathrm{SFr} / \mathrm{PFr}|>30)$
Regarding the models related to the signal processing, for example the one in charge to calculate the RMS value of the busbar residual voltage, the description in EMTP and Modelica are similar. However, with EMTP, it is defined according to a connection of some basic blocks while it is mathematically declared in Modelica (Figure 16). By the other, it should be denoted that, although the type of the input/output blocks in the two cases are causal, with Modelica, unlike EMTP, the integral equation is acausal. It is a very important feature of Modelica that allows the user to declare a system without worrying about the order of the equations.

\section{Figure 16 :Model providing the RMS value of} the busbar residual volatage

\section{Model under EMTP}

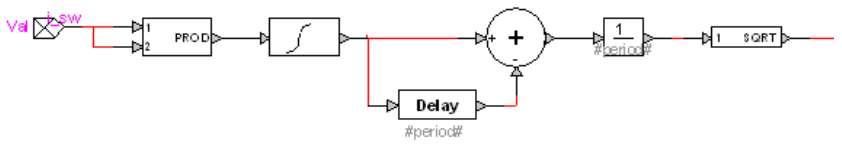

Modelica Model

Moreover, contrary to EMTP, it is important to underline that all Modelica components are not solver dependent. Under EMTP, both with a source code or a DLL, a $\mathrm{C}$ code program of the discretized equations of the system should be created according to the EMTP solver. In the opposite, this task is automatically performed by the symbolic solver in the Dymola tool. 


\section{Conclusions}

It is undeniable that the opening provided by Modelica allows an unmatched flexibility in modeling. Contrary to EMTP, the PWH model is very close to the description given in the paper specification. In other words, Modelica description corresponds to a formal version of the specification while under EMTP, given the basic blocks available, the model obtained is rather a translation of the specification.

In addition our study shows the great interest of the Modelica language for power electrical system modeling and for electrotechnical studies.

So, for EDF R\&D, the following steps will be :

- To use actual measurements as input to the PWH Modelica model in order to study more precisely the behavior of the protection,

- If required, to update the model and create a new release of the specification including the textual Modelica model.

For this work, one of the intended goals will be to examine the ModelicaML profile [2] as an opportunity to graphically specify a system behavior.

References :

[1]

Spécification Technique EDF

HN 45-S-54

Régime de neutre compensé -

Spécification de la Protection Wattmétrique Homopolaire

[2]

http://www.openmodelica.org/index. php/developer/tools/134

ModelicaML - a UML profile for Modelica

http://www.emtp.com/

EMTP-RV - Computational Engine

[4] Principles of Object-Oriented

Modeling and Simulation with

Modelica 2.1

Peter Fritzson 